\title{
Rhodobacter capsulatus genes encoding form I ribulose-1,5-bisphosphate carboxylase/oxygenase (cbbLS) and neighbouring genes were acquired by a horizontal gene transfer
}

\author{
George C. Paoli, ${ }^{1} \dagger$ Ferda Soyer, ${ }^{2}$ Jessup Shively ${ }^{2}$ and F. Robert Tabita'
}

Author for correspondence: F. Robert Tabita. Tel: +1614292 4297. Fax: +1 6142926337.

e-mail: Tabita.1@osu.edu

1 The Department of Microbiology and Plant Molecular Biology/ Biotechnology Program, The Ohio State University, 484 West 12th Avenue, Columbus, OH 43210-1292, USA

2 Department of Biological Sciences, Clemson University, Clemson, SC 29634, USA

\begin{abstract}
Analysis of the nucleotide sequence of the form I ribulose-1,5-bisphosphate carboxylase/oxygenase (RubisCO) genes (cbbL and cbbS) of the non-sulfur purple bacterium Rhodobacter capsulatus indicated that the deduced amino acid sequence of the large subunit was not closely homologous to the large subunit from related organisms. Indeed, phylogenetic analysis suggested that the large subunit protein (CbbL) more closely resembled the enzyme from $\alpha / \beta / \gamma$ purple bacteria and cyanobacteria and is within a 'green-like' radiation of the RubisCO phylogenetic tree, well separated from CbbL of the related organism Rhodobacter sphaeroides. A cbbQ gene was discovered downstream of cbbs in $\boldsymbol{R h}$. capsulatus, a gene arrangement which also appears to be limited to certain organisms containing a 'green-like' RubisCO. Upstream, and divergently transcribed from cbbLSQ, is a gene (cbbR) that encodes a LysR-type transcriptional activator. Phylogenetic analysis of the deduced amino acid sequence of CbbR, also suggests that this protein is quite distinct from the $R \boldsymbol{h}$. sphaeroides CbbR protein, and is even distinct from the previously described $\mathrm{CbbR}_{\mathrm{II}}$ protein, the gene of which is upstream and divergently transcribed from the $\mathbf{c b b}_{\text {, }}$ operon of $R$ h. capsulatus. Interestingly, Rh. capsulatus CbbR, is more closely related to CbbR from bacteria whose RubisCO falls within the 'greenlike' radiation of the CbbL tree. These studies suggest that the $c b b R_{1}-c b b L-c b b S-c b b Q$ genes were acquired by $R h$. capsulatus via horizontal gene transfer from a bacterial species containing a 'green-like' RubisCo.
\end{abstract}

Keywords: RubisCO, Rhodobacter capsulatus, phylogeny, $c b b R, c b b Q$

\section{INTRODUCTION}

Rhodobacter capsulatus, a non-sulfur purple photosynthetic bacterium, assimilates $\mathrm{CO}_{2}$ via the CalvinBenson-Bassham (CBB) reductive pentose phosphate pathway. The $\mathrm{CBB}$ enzyme responsible for fixing $\mathrm{CO}_{2}$ is ribulose-1,5-bisphosphate carboxylase/oxygenase (RubisCO), an enzyme which exists in two distinct forms. The form I RubisCO is a large hexadecameric

†Present address: Armstrong Laboratory, Tyndall Air Force Base, FL 32403, USA.

Abbreviation: RubisCO, ribulose-1,5-bisphosphate carboxylase/ oxygenase.

The GenBank accession numbers for the sequences reported in this paper are L82000 (cbbLSQ) and U87283 (cbbR). enzyme $\left(\mathrm{L}_{8} \mathrm{~S}_{8}\right)$ composed of eight large subunits $\left(M_{\mathrm{r}} \sim\right.$ $55000)$ and eight small subunits $\left(M_{\mathrm{r}} \sim 15000\right)$ which in proteobacteria are encoded by the $c b b L$ and $c b b S$ genes, respectively. The form II RubisCO, encoded by the cbbM gene (Gibson, 1995), is composed of large subunits only $\left(\mathrm{L}_{x}\right)$; these are biochemically and genetically distinct from form I large subunits. The non-sulfur purple photosynthetic bacterium Rhodobacter sphaeroides was the first organism shown to synthesize both forms of RubisCO (Gibson \& Tabita, 1977a) and the organization and regulation of the genes encoding CBB enzymes ( $c b b$ genes) have been intensely studied in $R h$. sphaeroides and other autotrophic bacteria (Gibson \& Tabita, 1996).

The organization of the $c b b$ genes in the closely related 
organism Rh. capsulatus was determined recently (Paoli et al., 1995). The Rh. capsulatus form II RubisCO gene, $c b b M$, has been sequenced (Larimer et al., 1995) and found to encode a protein that is very similar to the form II RubisCO from $R h$. sphaeroides. As for the form I enzyme, earlier studies had indicated that the $R h$. capsulatus form I RubisCO exhibited properties that were distinct from its counterpart in $R h$. sphaeroides, as noted by differences in its elution behaviour from anionexchange columns and by differences in antibody crossreactivity (Gibson \& Tabita, 1977b). More recently, recombinant $R h$. capsulatus form I Rubis CO was shown to cross-react with antibodies directed against cyanobacterial RubisCO, with little or no reactivity to anti$R h$. sphaeroides form I RubisCO in Western blot experiments (Paoli et al., 1995). In the hope of gaining a better understanding of its unusual properties, the current study was undertaken to determine the primary structure of the $R h$. capsulatus form I RubisCO as well as upstream and downstream sequences.

The unique $c b b$ gene organization and subsequent phylogenetic analyses of the $R h$. capsulatus CbbL and putative upstream transcriptional activator $\mathrm{CbbR}_{\mathrm{I}}$ indicated that the genes encoding $R h$. capsulatus form I RubisCO and neighbouring genes were acquired by a horizontal gene transfer.

\section{METHODS}

DNA sequencing and analysis. The sequence of the $R h$. capsulatus $c b b L S Q$ genes from cosmid pRCFI was determined by automated sequencing using an $\mathrm{ABI} 377 \mathrm{a}$ DNA sequencer with ABI Taq DyeDeoxy Cycle sequencing reagents and a Perkin-Elmer Cetus thermal cycler, as described by PerkinElmer. Plasmids pRKFIP, pK18FISB, pK18FI5EH, pK18FI6E, pK18FI7EH, pULHSm531 and pULHSm320 (Table 1) were used, and, as necessary, oligonucleotide primer walking was employed to generate complete double-stranded sequence. Synthetic oligonucleotides were purchased from Integrated DNA Technologies. Because of the unusual nature of the $R h$. capsulatus cbbLS sequence, the identity of the $c b b L$ gene was confirmed from partial sequencing of clones derived from two additional sources (pEULA4 and pK1H2E7; Fig. 1, Table 1). The nucleotide sequence of $R b$. capsulatus $c b b R_{\mathrm{I}}$ and $c b b L$, from plasmids pEULA4 and PK1H2E7, was determined using an ABI Prism 310 Genetic Analyzer. The M13/pUC forward 23 base sequencing primer $\left(5^{\prime}\right.$-CCCAGTCACGACGTTGTAAAACG- $3^{\prime}$ ), M13 reverse $(-48)$ primer ( $5^{\prime}$-AGCGGATAACAATTTCACACAGGA-3') and sequence-specific synthetic primers were used to complete the double-stranded sequence. Sequences were analysed using the University of Wisconsin Genetics Computing Group (GCG) software, the EGCG Extension Programs (The Sanger Centre, Hinxton, England) and MacVector Sequence Analysis software (International Biotechnology, Inc., New Haven, CT, USA).

Phylogenetic analysis of $R h$. capsulatus RubisCO and CbbR protein sequences. Multiple sequence alignments were performed using CLUSTAL w (Thompson et al., 1994) and trees were generated by neighbour-joining and maximum parsimony methods using PHYLIP 3.5c (Felsenstein, 1989) and by maximum likelihood using Puzzle 2.5.1 (Strimmer \& von Haeseler, 1996). Accession numbers (SWISS-PROT or GenBank databases) of sequences used for phylogenetic analysis of form I RubisCO large subunit proteins (CbbL or $\mathrm{RbcL}$ ) are as follows: Anabaena sp. PCC 7120, P00879; Chlamydomonas reinhardtii, P00877; Chromatium vinosum

Table 1. Plasmids used in this study

\begin{tabular}{|c|c|c|}
\hline Plasmid & Relevant characteristics & Source/reference \\
\hline pK18, pK19 & $\mathrm{Km}^{\mathrm{R}}$; pUC derivatives & Pridmore (1987) \\
\hline pRKFIP & $\begin{array}{l}\text { pRK } 415 \text { containing the } 9 \mathrm{~kb} \text { Pst I fragment of pRCFI } \\
\text { containing the } R h . \text { capsulatus cbbLS genes }\end{array}$ & Paoli et al. (1995) \\
\hline pK18FISB & $\begin{array}{l}\text { pK18 containing part of the } R h . \text { capsulatus } c b b L \text { gene on a } \\
1.8 \mathrm{~kb} \mathrm{BamHI}-\text { Sall fragment cloned from pRKFIP }\end{array}$ & This work \\
\hline pK18FI5EH & $\begin{array}{l}\text { pK18 containing part of the } R h . \text { capsulatus } c b b L \text { gene on a } \\
0.5 \mathrm{~kb} \text { HindIII-EcoRI fragment cloned from pK18FISB }\end{array}$ & This work \\
\hline pK18FI6E & $\begin{array}{l}\text { pK18 containing part of the } R h . \text { capsulatus } c b b L \text { gene on a } \\
0.6 \mathrm{~kb} \text { EcoRI fragment cloned from pK18FISB }\end{array}$ & This work \\
\hline pK18FI7EH & $\begin{array}{l}\text { pK18 containing part of the } R h . \text { capsulatus } c b b L \text { gene on a } \\
0.7 \mathrm{~kb} \text { EcoRI-HindIII fragment cloned from pK18FISB }\end{array}$ & This work \\
\hline pEULA4 & $\begin{array}{l}\text { pK19 containing part of the } R b . \text { capsulatus } c b b L \text { gene and } \\
\text { upstream sequence on a } 4 \mathrm{~kb} \text { EcoRI fragment }\end{array}$ & This work \\
\hline pULHSm531 & $\begin{array}{l}\text { pK19 containing part of the } R h . \text { capsulatus } c b b L \text { gene and } \\
\text { upstream sequence on a } 0.5 \mathrm{~kb} \text { HindIII-Smal fragment } \\
\text { cloned from pEULA4 }\end{array}$ & This work \\
\hline pULHSm 320 & $\begin{array}{l}\text { pK19 containing part of the } R h \text {. capsulatus } c b b R_{\mathrm{I}} \\
\text { sequence on a } 0.36 \mathrm{~kb} \text { HindIII-Smal fragment cloned } \\
\text { from pEULA } 4\end{array}$ & This work \\
\hline $\mathrm{p} 1 \mathrm{H} 2$ & $\begin{array}{l}\text { A } c b b L \text {-hybridizing clone derived from an } R h . \text { capsulatus } \\
\text { SB1003 overlapping cosmid library }\end{array}$ & Fonstein et al. (1993) \\
\hline pK1H2E7 & $\begin{array}{l}\text { pK18 containing part of the } R b \text {. capsulatus } c b b L \text { gene } \\
\text { sequence on a } 0.7 \mathrm{~kb} \text { EcoRI fragment cloned from p1H2 }\end{array}$ & This work \\
\hline
\end{tabular}


1, P22849; Chromatium vinosum 2, P22859; Cryptomonas $\Phi$, P14957; Cyanophora paradoxa, P24312; Cuscuta reflexa, P30401; Cylindrotheca sp. N1, P24673; Euglena gracilis, P00878; Hydrogenovibrio marinus 1, D43621; Hydrogenovibrio marinus 2, D43622; Ipomoea purpurea, P28260; Mollugo verticillata, P25832; Nicotiana tabacum, P00876; Nitrobacter vulgaris, L22885; Odontella sinensis, P49520; Olisthodiscus luteus, P14959; Porphyra purpurea, P50255; Prochlorococcus marinus, D21833; Prochloron sp., D21834; Prochlorothrix hollandica, P27586; Pseudomonas hydrogenothermophila, D30764; $R h$. capsulatus, L8200; $R h$. sphaeroides, P27997; Ralstonia eutropha ATCC 17707 (chromosomal), M17744; Ralstonia eutropha ATCC 17699 (chromosomal), P09657; Ralstonia eutropha ATCC 17699 (plasmid), U20585; Spinacia oleracea, P00875; Synechococcus sp. PCC 6301, P00880; Synechococcus sp. PCC 7002, D13971; Synechococcus sp. WH7803, U46156; Thiobacillus denitrificans, L42920; Thiobacillus ferrooxidans, P28895; Vent worm (Alvinoconcha) symbiont (potential Thiobacillus sp.), P24672; Triticum aestivum, P11383; Xanthobacter flavus, P23011.

Accession numbers of sequences used for phylogenetic analysis of form II RubisCO large subunit proteins (CbbM or RbcL) are as follows: Gonyaulax polyedra, LL41063; Hydrogenovibrio marinus, D28135; $R h$. capsulatus, P50922; Rh. sphaeroides, P29278; Rbodospirillum rubrum, P04718; Symbiodinium sp., U43532; T. denitrificans, L37437.

Accession numbers of sequences used for phylogenetic analysis of CbbR proteins are as follows: Chr. vinosum (CbbR), P25544; Cyanophora paradoxa (plastid) (YCF30/RbcR), P48271; Odontella sinensis (plastid) (YCF30/RbcR), P49518; Porphyra purpurea (plastid) (YCF30/RbcR), P51205; Ra. eutropha (CbbR), P42722; Rh. capsulatus (AmpR), P14145; $R h$. capsulatus (CbbR $\left.{ }_{\mathrm{I}}\right), \mathrm{U} 87283 ; R h$. capsulatus $\left(\mathrm{CbbR}_{\mathrm{II}}\right)$, U87282; Rh. sphaeroides (CbbR), P52690; Rs. rubrum (CbbR), P52595; Synechocystis sp. PCC 6803 (RbcR1), CyanoBase ORF ID = SLL0030; Synechocystis sp. PCC 6803 (RbcR2), CyanoBase ORF ID = SLL1594; T. ferrooxidans (CbbR), Q06610; X. flavus (CbbR), P25545.

\section{RESULTS}

\section{Organization of the Rh. capsulatus cbbLS genes and neighbouring regions}

In a previous study, gene-specific probes and a $c b b L$ hybridizing $R h$. capsulatus library clone indicated that the $R h$. capsulatus cbbLS genes, encoding form I RubisCO, were not associated with any other known $c b b$ structural genes (Paoli et al., 1995). DNA sequence analysis of subclones derived from $c b b L S$ containing cosmid pRCFI revealed that the $31 \mathrm{~kb}$ chromosomal DNA insert in this library clone contained only $100 \mathrm{bp}$ $R h$. capsulatus DNA upstream of the $c b b L$ gene. A $4 \mathrm{~kb}$ EcoRI fragment containing part of the $R h$. capsulatus $c b b L$ gene and upstream DNA was cloned from the $R h$. capsulatus SB1003 chromosome into pK19 (pEULA4; Fig. 1) to determine if other $c b b$ genes were present upstream of the form I RubisCO genes. The organization of $c b b L S$ and the neighbouring region is presented in Fig. 1. Nucleotide sequencing revealed an open reading frame (ORF) with a deduced amino acid sequence similar to the CbbR class of LysR-type transcriptional activators upstream and divergently transcribed from $c b b L$; in addition, the previously identified anfA gene (Kutsche et al., 1996) was found to be about $2 \mathrm{~kb}$ downstream of $c b b R_{\mathrm{I}}$. The subscript I indicates that the divergently transcribed $c b b R_{\mathrm{I}}$ gene is associated with the form I RubisCO genes. A second $c b b R$ gene, $c b b R_{\mathrm{II}}$, is present upstream and divergently transcribed from a group of $c b b$ genes associated with the form II RubisCO gene of Rh. capsulatus (Paoli et al., 1995). Additional nucleotide sequencing indicated that no other known $c b b$ genes were present in the region between $c b b R_{\mathrm{I}}$ and anf $A$ (J. L. Gibson, unpublished). Sequencing downstream of $c b b S$ revealed an ORF with a predicted amino acid sequence having significant similarity to the $c b b Q$ gene product from Ps. bydrogenothermophila (Yokoyama et al., 1995).

\section{Nucleotide sequence of $c b b R_{1}, c b b L, c b b S$ and $c b b Q$}

The nucleotide sequence for both strands of a 3993 nucleotide region including the form I RubisCO genes and approximately $1 \mathrm{~kb}$ downstream (GenBank accession no. L82000) and approximately $1 \mathrm{~kb}$ upstream (GenBank accession no. U87283) of cbbLS was determined. An ORF of 897 nucleotides, 110 nucleotides upstream of $c b b L$ and divergently transcribed from $c b b L$, extends from an ATG start codon at position 948 to a TGA stop codon at position 52. The ORF is preceded by a potential ribosome-binding site, GGA, 11 bp upstream of the putative start codon. The coding region is 298 amino acids long with a deduced $M_{\mathrm{r}}$ of 31766. A database search for proteins with amino acid homology revealed that the product of this ORF is most similar to members of the CbbR family of LysR-type transcriptional activators. In a number of autotrophic bacteria (Gibson \& Tabita, 1996), $c b b R$ is divergently transcribed from the $c b b$ genes and in several cases CbbR has been shown to activate transcription of the associated $c b b$ genes (Gibson \& Tabita, 1993; Kusano \& Sugawara, 1993; van den Bergh et al., 1993; Viale et al., 1991; Windhovel \& Bowien, 1991). Recently, genome sequencing projects have revealed ORFs whose products have homology to CbbR, including those in the cyanobacterium Synechocystis sp. PCC 6803, the plastids of the non-green algae Odontella sinensis and Porphyra purpurea, and the plastids of the glaucophyte Cyanophora paradoxa. In addition, $R h$. capsulatus $\mathrm{CbbR}_{\mathrm{I}}$ shares $51.0 \%$ deduced amino acid sequence identity with CbbR from $T$. ferrooxidans, but only $42.7 \%$ identity with $R h$. capsulatus $\mathrm{CbbR}_{\mathrm{II}}$, and $37.2 \%$ identity with $R h$. sphaeroides CbbR.

$R h$. capsulatus cbbL starts at position 1058 and is preceded by a ribosome-binding site five nucleotides upstream of the ATG start codon. A T- $\mathrm{N}_{11}$-A motif has been identified as a common feature of the binding site for LysR-type transcriptional activators (Goethals et al., 1992); this motif usually has a 3 bp inverted repeat at each end and is often repeated two or three times upstream of the regulated gene or operon. Three such $T$ $\mathrm{N}_{11}$-A motifs were found in the $c b b R_{\mathrm{I}}-c b b L$ intergenic region. The $c b b L$ gene was shown to be 1422 nucleotides in length and to encode a protein of 473 amino acids 


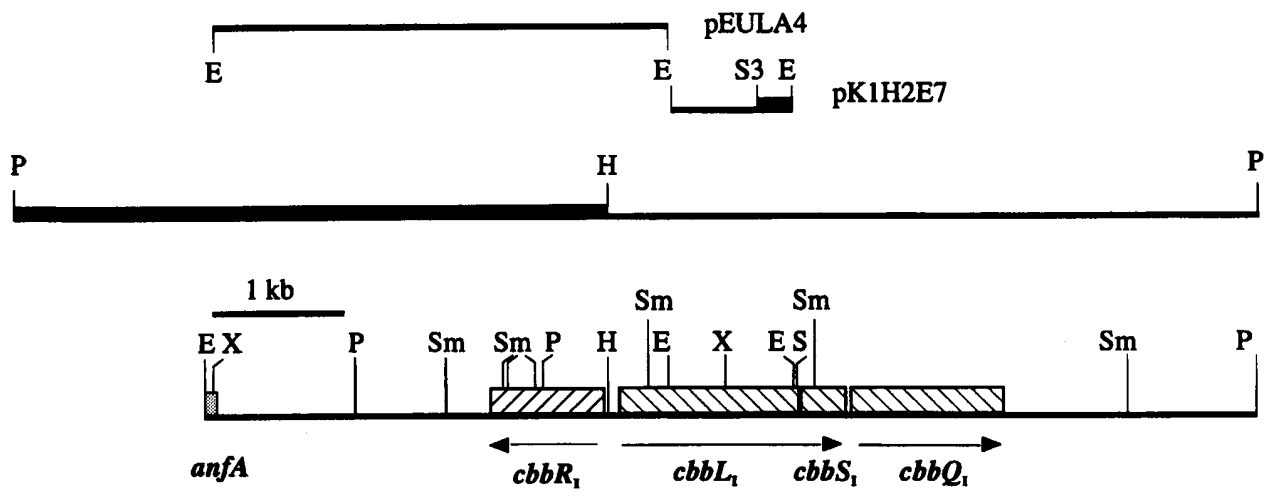

Fig. 1. Genetic and physical map of the Rh. capsulatus $c b b_{1}$ gene cluster. Arrows below the genes indicate the size and direction of potential transcripts. Restriction enzymes sites: E, EcoRI; H, HindIII; P, Pstl; S, Sall; Sm, Smal; X, Xhol. The thicker lines represent cosmid DNA that was subcloned along with the $R h$. capsulatus chromosomal DNA in the original library clones. Only $100 \mathrm{bp} R h$. capsulatus chromosomal DNA is present upstream of the cbbL gene in pRKFIP. pEULA4 is a $4 \mathrm{~kb}$ EcoRI fragment cloned directly from $R h$. capsulatus chromosomal DNA. pK1H2E7 contains a 700 bp EcoRI fragment subcloned from cosmid p1H2 (Fonstein et al., 1993). As indicated, the insert in plasmid pK1H2E7 is in part cosmid DNA, because cosmid $\mathrm{p} 1 \mathrm{H} 2$ did not contain the entire cbbL gene. The Sau3A (S3) site at the end of the Rh. capsulatus chromosomal DNA insert in p1H2 is shown.

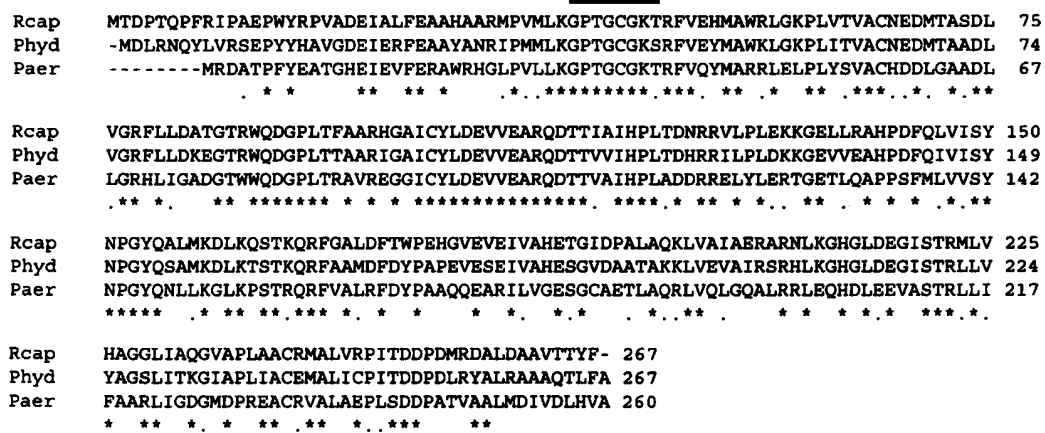

Fig. 2. CbbQ amino acid sequence alignment. Amino acid alignment of the presumptive CbbQ of $R h$. capsulatus (Rcap) compared to CbbQ of Ps. hydrogenothermophila (Phyd) and Ps. aeruginosa NirQ (Paer). The putative P-loop nucleotide-binding motif [(G/A) $\left.\mathrm{X}_{4} \mathrm{GK}(\mathrm{S} / \mathrm{T})\right]$ (Walker et al., 1982) is indicated by the dark bar over the alignment.

with a predicted $M_{\mathrm{r}}$ of 53036 . The highest sequence identity $(86.0 \%)$ was found with CbbL of Ps. bydrogenothermophila. Significantly less identity $(57.8 \%)$ was noted when the $R h$. capsulatus CbbL was compared to the $R h$. sphaeroides form I RubisCO large subunit. This is particularly interesting in light of earlier reports which indicated that the $R h$. capsulatus form I RubisCO was immunologically distinct from that of the closely related organism $R h$. sphaeroides (Gibson \& Tabita, 1977b; Paoli et al., 1995). The $c b b L$ sequence was obtained from subclones of pRCFI (Table 1, Fig. 1), a cosmid clone from an $R h$. capsulatus SB1003 chromosomal DNA library (Scolnik \& Haselkorn, 1984). Because of the unusual sequence of the $c b b L$ gene, the sequence was confirmed by analysing clones from two additional sources. Portions of the $c b b L$ gene present on the $R h$. capsulatus SB1003 chromosomal DNA fragment in plasmid pEULA4 (Table 1, Fig. 1) and a subclone (pK1H2E7; Fig. 1, Table 1) from a cosmid clone obtained from an $R h$. capsulatus SB1003 overlapping cosmid library (Fonstein et al., 1993) were also sequenced. The sequence derived from these clones was identical to the sequence obtained from the pRCFI subclones.
An ORF encoding the $R h$. capsulatus form I RubisCO small subunit starts at an ATG codon 22 nucleotides downstream of the $c b b L$ stop codon and a potential ribosome-binding site was present six nucleotides upstream of the $c b b S$ start codon. The $R h$. capsulatus CbbS protein was predicted to be 118 amino acids in length, with an $M_{r}$ of 13548 . The highest sequence identity, $67 \cdot 8 \%$, was to $T$. denitrificans CbbS. The Ps. bydrogenothermophila CbbS was $60.7 \%$ identical and CbbS from the closely related organism $R h$. sphaeroides shared only $33.0 \%$ identity. Two inverted repeat sequences that might function in transcriptional termination were found downstream of $c b b S$. Further downstream of $c b b S$ was an ORF predicted to encode a protein of 267 amino acids with an $M_{r}$ of 29429 . The putative ATG codon at position 2958 was 100 nucleotides downstream of the $c b b S$ stop codon and preceded by a plausible ribosome-binding site. The deduced amino acid sequence of the product of this ORF was $72.0 \%$ identical to CbbQ of Ps. hydrogenothermophila and $52.3 \%$ identical to Pseudomonas aeruginosa NirQ. Alignment of the predicted amino acid sequence of the $R h$. capsulatus CbbQ with the Ps. hydrogenothermophila CbbQ and Ps. aeruginosa NirQ (Fig. 2) revealed 


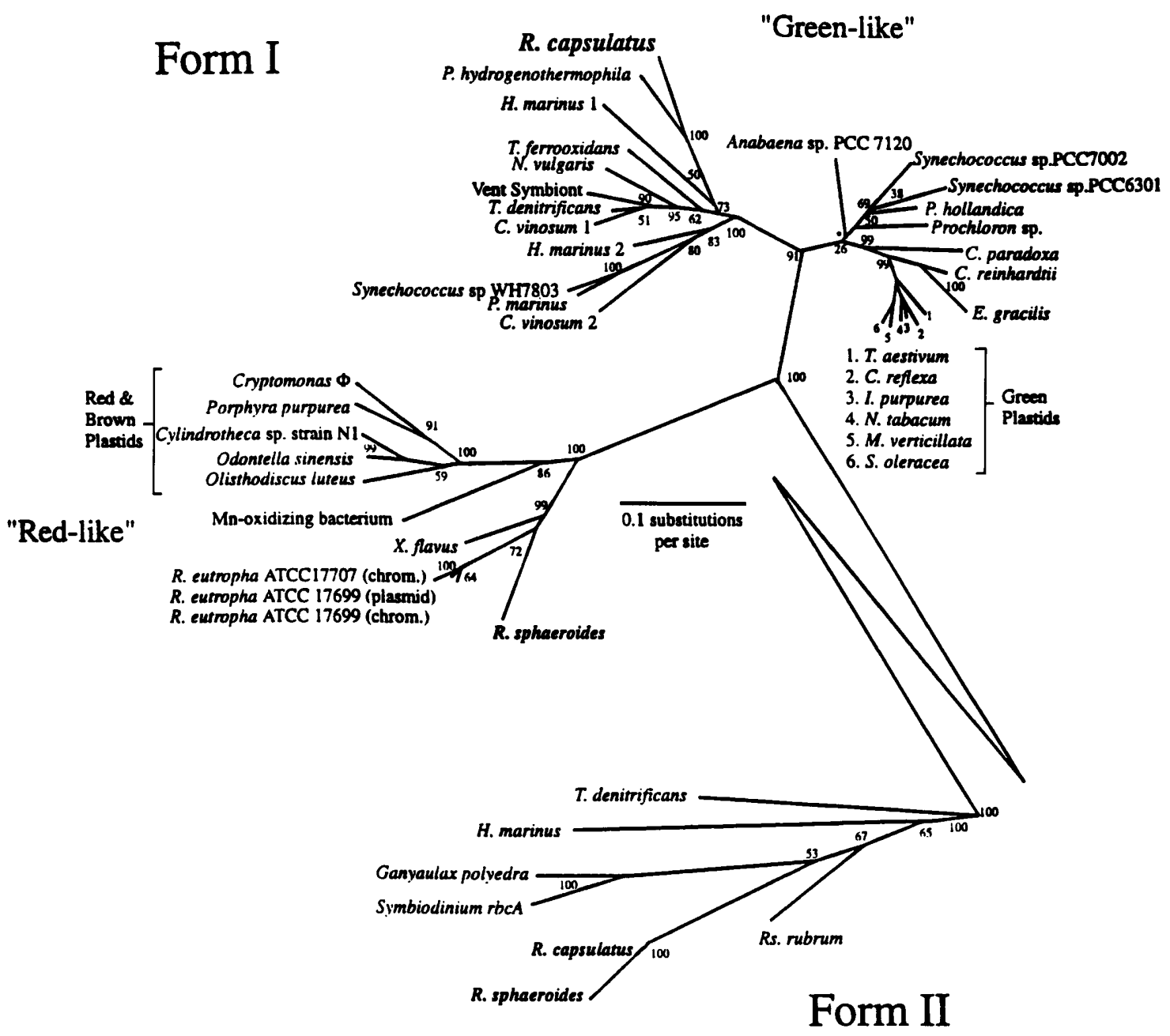

Fig. 3. RubisCO large subunit phylogenetic tree. Amino acid sequence alignments were performed using CLUSTAL W. The 412-amino-acid alignment corresponds to the sequence of $R h$. capsulatus CbbL from W59 to T454. The tree was generated by neighbour-joining (Kimura distances) using the Protdist and Neighbor programs of PHYLIP 3.5. Bootstrap values were calculated from 1000 replicates of the Seqboot, Protdist, Neighbor and Consense programs of PHYLIP 3.5, and are indicated at the nodes as percentages. This tree is unrooted.

several regions of sequence identity, including a putative ATP-binding domain. Interestingly, two T- $\mathrm{N}_{11}$-A LysRbinding motifs were present within the $c b b S-c b b Q$ intergenic region.

\section{Phylogenetic analysis of the $R h$. capsulatus form I RubisCO large subunit}

Previous work had indicated that the $R h$. capsulatus form I RubisCO exhibited anomalous properties; these findings, combined with the unexpected sequence identity to $\mathrm{CbbL}$ of Ps. hydrogenothermophila and the relatively poor sequence identity to $R h$. sphaeroides CbbL, prompted a phylogenetic analysis of the $R h$. capsulatus CbbL. Phylogenetic analysis of a broad range of RubisCO large subunits was conducted using the neighbour-joining method (Fig. 3). The tree is modified from one presented previously (Watson \& Tabita, 1997) in that Symbiodinium sp. RbcG has been excluded and the $R b$. capsulatus, Porphyra purpurea and Odontella sinensis form I RubisCO large subunit sequences have been included. Both form I and form II RubisCO were included in this analysis and the form I RubisCO 'redlike' and 'green-like' classification of Delwiche $\&$ Palmer (1996) was retained. Despite the close phylogenetic relationship between $R h$. capsulatus and $R h$. sphaeroides based on 16S rRNA sequences (Woese et al., 1984), and the phylogenetic relatedness of the $R h$. capsulatus and $R h$. sphaeroides form II RubisCO large subunits (Fig. 3), the RubisCO phylogenetic tree indicates that the form I RubisCO large subunits from these two organisms are not closely related. $R h$. capsulatus CbbL is within a 'green-like' radiation containing species of $\alpha / \beta / \gamma$ purple bacteria and cyanobacterial species. The $R h$. capsulatus sequence shares a branch with the sequence from Ps. hydrogenothermophila, the protein most similar based on comparisons of deduced amino acid sequences. In contrast, the $R h$. sphaeroides CbbL is within the 'red-like' radiation. Bootstrap values are indicated and strongly support the 


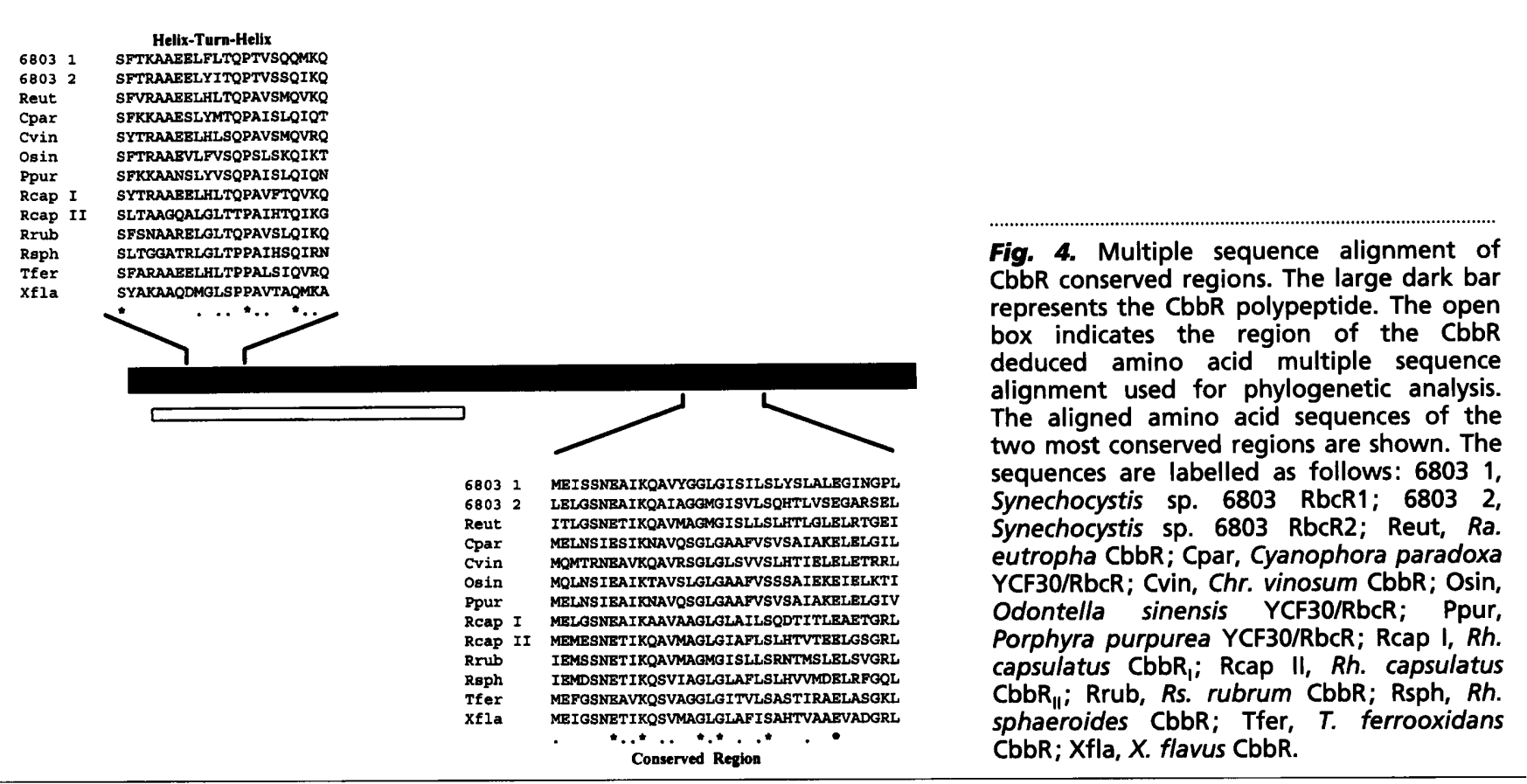

tree topology. Trees with similar topology were generated by maximum parsimony and maximum likelihood analysis.

\section{Phylogenetic analysis of CbbR transcriptional activators}

Like the $R h$. capsulatus form I RubisCO large subunit, the $\mathrm{CbbR}_{\mathrm{I}}$ sequence showed a significantly lower similarity to $R h$. sphaeroides CbbR than to CbbR from more distantly related organisms. Thus, the possibility that $R h$. capsulatus $\mathrm{CbbR}_{\mathrm{I}}$ has a similar phylogeny to that of the $R h$. capsulatus form I RubisCO was investigated.

A phylogenetic analysis of 50 LysR-type transcriptional activators revealed that the CbbR class of LysR-type proteins grouped together (not shown). Analysis of the LysR-type proteins was carried out using the $\mathrm{N}$-terminal region of the amino acid sequence which includes the functionally conserved helix-turn-helix DNA-binding domain. The region of the amino acid sequences used for tree building was a 145 residue sequence alignment generated by CLUSTAL W (Fig. 4). Alignment of amino acid sequences of 50 LysR-type proteins revealed no absolutely conserved residues within this region and only four residues that were represented by conservative substitutions (data not shown). A multiple sequence alignment of the CbbR deduced amino acid sequences revealed additional sequence similarity within this class of LysR-type transcriptional activator (Fig. 4). Most of the sequence similarity was limited to the helix-turnhelix motif and a second relatively conserved region of 36 residues corresponding to a previously recognized Cterminal domain (Schell, 1993). The C-terminal domain is thought to be involved in ligand binding or multimerization required for DNA binding (Schell, 1993).

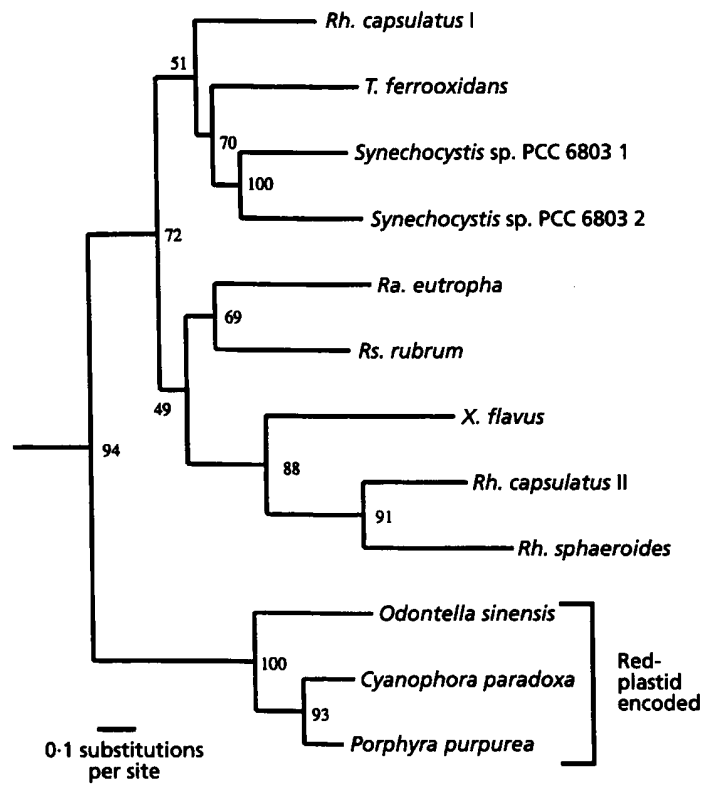

Fig. 5. CbbR phylogenetic tree. A multiple sequence alignment of 145 amino acids [corresponding to the region of the CbbR polypeptide indicated by the open box (Fig. 4), amino acids Q7-G148 of $R h$. capsulatus CbbR $]$ was generated by CLUSTAL W. The CbbR tree and bootstrap values were produced by the methods detailed in the legend to Fig. 3. The tree was rooted using $R h$. capsulatus AmpR. The relationship of Chr. vinosum CbbR to the other CbbR proteins varied when different phylogenetic analyses were used. It was thus excluded from the analysis presented to prevent giving undue weight to a single topology.

The results of a neighbour-joining analysis of CbbR proteins (Fig. 5) shows the phylogenetic relationship between $R h$. capsulatus $\mathrm{CbbR}_{\mathrm{I}}$ and $T$. ferrooxidans 
CbbR in addition to the more distant branching of the $R h$. sphaeroides CbbR, which is itself closely related to $R$ b. capsulatus $\mathrm{CbbR}_{\mathrm{II}}$. This tree is based on the CLUSTAL $w$ alignment of the $\mathrm{N}$-terminal region and trees with identical topology were generated from an alignment of the complete CbbR sequences. In addition, maximum parsimony and maximum likelihood analyses generated trees of the same topology. The CbbR phylogenetic tree (Fig. 5) is in good agreement with the RubisCO phylogenetic tree (Fig. 3) in that $R$ h. capsulatus $\mathrm{CbbR}_{\mathrm{I}}$ is on a branch with CbbR from the 'green-like' RubisCOcontaining bacterium $T$. ferrooxidans and the cyanobacterium Synechocystis sp. strain PCC 6803. It should be pointed out that the two CbbR homologues from Synechocystis sp. strain PCC 6803, and those encoded by red plastid genomes, are not transcribed divergently from $c b b$ genes; thus, their classification as CbbR/RbcR proteins is based solely on the similarity of their deduced amino acid sequences to other CbbR proteins. The deep branching of the Synechocystis RbcR proteins (Fig. 5) suggests that they may well be CbbR homologues, despite the fact that neither of the genes which encode these proteins is divergently transcribed from $c b b$ genes. In contrast, the red-plastid-encoded putative $\mathrm{RbcR}$ proteins form a separate branch in the CbbR tree. In fact, the red plastid $\mathrm{RbcR}$ sequences grouped within the CbbR family branch of the neighbour-joining and maximum likelihood LysR trees, but fell outside the CbbR family branch in a maximum parsimony LysR tree (not shown). Thus, the phylogenetic association of the red plastid $\mathrm{RbcR}$ proteins with the other CbbR proteins is tentative.

\section{DISCUSSION}

The nucleotide sequence of the genes encoding the $R h$. capsulatus form I RubisCO was determined and additional $c b b$ genes were found upstream and downstream from $c b b L S$. The $c b b R_{\text {I }}$ gene was found upstream and divergently transcribed from $c b b L$, and is the second $c b b R$ gene found in $R b$. capsulatus. The other $c b b R$ gene, now designated $c b b R_{\mathrm{II}}$, is upstream and divergently transcribed from the $R h$. capsulatus $c b b_{\text {II }}$ operon (Paoli et al., 1995). T. denitrificans has two $c b b R$ genes (Lorbach \& Shively, 1995) and, in this organism, $c b b R_{\mathrm{I}}$ and $c b b R_{\mathrm{II}}$ are directly upstream and divergently transcribed from the $c b b L S$ and $c b b M$ genes, respectively. More detailed experiments have shown that a single CbbR regulates the expression of at least two $c b b$ operons in $R h$. sphaeroides (Gibson \& Tabita, 1993) and the chromosomal and plasmid $c b b$ operons of Alcaligenes eutrophus (now called Ralstonia eutropha) (Windhovel \& Bowien, 1991). In X. flavus, CbbR is required for the regulated expression of the $c b b$ operon (van den Bergh et al., 1993) and at least one other operon (Meijer et al., 1996). The presence of two CbbR proteins in $R h$. capsulatus could allow independent regulation of the $c b b$ operons of this organism. If $\mathrm{CbbR}_{\mathrm{I}}$ and $\mathrm{CbbR}_{\mathrm{II}}$ bind different inducer molecules, these proteins might allow the expression of the two $c b b$ operons to be regulated by different environmental or metabolic sig- nals. Thus, it will be interesting to determine the role of each $\mathrm{CbbR}$ in $c b b$ gene expression in $R h$. capsulatus.

A gene encoding an apparent CbbQ protein was found downstream of $c b b S$ in $R h$. capsulatus. Likewise, the $c b b Q$ gene is immediately downstream from $c b b S$ in both Ps. hydrogenothermophila and Chr. vinosum (Yokoyama et al., 1995). CbbQ shares considerable amino acid homology with the NirQ protein of Pseudomonas spp. (Yokoyama et al., 1995; Fig. 2). The nirQ genes of Ps. aeruginosa (Arai et al., 1994) and Pseudomonas stutzeri (Jungst \& Zumft, 1992) are within a cluster of genes encoding proteins required for nitrite respiration. A strain of Ps. stutzeri in which nirQ had been disrupted lacked nitrite reductase and nitric oxide reductase activity, despite the fact that the proteins were still synthesized, suggesting that NirQ may be involved in post-translational assembly or modification of the nir gene products (Jungst \& Zumft, 1992). A function has not yet been assigned to CbbQ, but preliminary investigations indicate that it may be involved in the posttranslational activation of RubisCO (Igarashi \& Kodama, 1996). In this respect, the association of the $c b b Q$ and $c b b L S$ genes in $R h$. capsulatus is very interesting. Since it is feasible to construct genetic knockout strains of $R h$. capsulatus the function of CbbQ may eventually be discerned.

The assertion that $R h$. capsulatus form I RubisCO was acquired by horizontal gene transfer is not the first suggestion that such events have taken place. The incongruence of the RubisCO phylogenetic tree with the 16S rRNA phylogenetic tree necessitates at least three (now four) horizontal transfers within the proteobacteria (Delwiche \& Palmer, 1996; Watson \& Tabita, $1996,1997)$. However, the evidence that $R h$. capsulatus form I RubisCO was acquired by such a mechanism is far more compelling than for any other presumed bacterial RubisCO gene transfer event. $R h$. sphaeroides and $R h$. capsulatus have been grouped in the same genus based on classical morphological and chemotaxonomic properties (Imhoff et al., 1984). These two species have been found to be closely related based on phylogenetic analysis of their 16S rRNA (Woese et al., 1984), 5S rRNA (Boulygina et al., 1993), 23S rRNA (Ludwig et al., 1995), cytochrome $c$ (Dickerson, 1980; Woese et al., 1980 ) and reaction centre $L$ and $M$ proteins (Blankenship, 1992). Even the form II RubisCO amino acid sequences of $R h$. capsulatus and $R h$. sphaeroides are more than $94 \%$ identical and show a close phylogenetic relatedness. Nevertheless, the form I RubisCO proteins from these two organisms are very distantly related (Fig. $3)$.

The phylogenetic analysis of the $R h$. capsulatus form I RubisCO is further supported by its unusual immunological properties. The enzyme cross-reacts poorly with antibody raised against $R h$. sphaeroides form I RubisCO (Gibson \& Tabita, 1977b; Paoli et al., 1995) but crossreacts well with antibody raised against the Synechococcus sp. 6301 RubisCO (Paoli et al., 1995). In addition, several years ago, J. L. Gibson, W. B. Whitman \& F. R. 
Tabita (unpublished results) examined cell extracts from several species of non-sulfur purple photosynthetic bacteria (Rhodocyclus tenuis, Rhodospirillum molischianum, Rhodopseudomonas palustris, Rhodomicrobium vaniellii, $R h$. sphaeroides and $R h$. capsulatus) by Ouchterlony immunodiffusion. They found that antibody raised against the $R h$. sphaeroides form I RubisCO cross-reacted with extracts from every species except $R h$. capsulatus. Thus, $R h$. capsulatus may be unique amongst the non-sulfur purple photosynthetic bacteria in having a 'green-like' form I RubisCO. On the strength of this evidence, it would seem that the $R h$. capsulatus form I RubisCO was acquired by a horizontal gene transfer from a bacterial species containing a 'greenlike' RubisCO. Although the evidence is not as compelling, phylogenetic analysis of the CbbR deduced amino acid sequences (Fig. 5) suggests that $R h$. capsulatus $\mathrm{CbbR}_{\mathrm{I}}$ may also have been acquired from an organism containing a 'green-like' RubisCO. In addition, $R h$. capsulatus RubisCO is most closely related to the RubisCO of Ps. bydrogenothermophila, which also has a $c b b Q$ gene directly downstream of its $c b b S$ gene (Yokoyama et al., 1995). The only other species known to have a $c b b Q$ gene directly downstream of cbbS is Chr. vinosum (Yokoyama et al., 1995) and the $\mathrm{Cbr}$. vinosum RubisCO falls within the same 'greenlike' radiation as $R h$. capsulatus and Ps. hydrogenothermophila. Thus, it seems likely that the $c b b R_{\mathrm{I}}, c b b L S$ and $c b b Q$ genes of $R b$. capsulatus were acquired by a single gene transfer event from some as yet unidentified bacterial species whose RubisCO falls within the bacterial/cyanobacterial 'green-like' RubisCO radiation (Fig. 4).

\section{ACKNOWLEDGEMENTS}

The authors would like to thank Dr Gregory Watson for his considerable help with the phylogenetic analysis, and Drs Michael Fonstein and Robert Haselkorn for providing the $c b b L$-hybridizing cosmid $\mathrm{p} 1 \mathrm{H} 2$ from their $R h$. capsulatus SB1003 overlapping cosmid library. This work was supported by USDA grant 92-37307-7663, grant MCB-9513481 from NSF (both to J.S.) and Public Health Service grant GM24497 from $\mathrm{NIH}$ (to F.R.T.).

\section{REFERENCES}

Arai, H., Igarashi, Y. \& Kodama, T. (1994). Structure and ANRdependent transcription of the nir genes for denitrification from Pseudomonas aeruginosa. Biosci Biotechnol Biochem 58, 1286-1291.

van den Bergh, E. R. E., Dijkhuizen, L. \& Meijer, W. G. (1993). CbbR, a LysR-type transcriptional activator, is required for expression of the autotrophic $\mathrm{CO}_{2}$ fixation enzymes of Xanthobacter flavus. J Bacteriol 175, 6097-6104.

Blankenship, R. B. (1992). Origin and early evolution of photosynthesis. Photosynth Res 33, 91-111.

Boulygina, E. S., Chumakov, K. M. \& Netrusov, A. L. (1993). Systematics of Gram-negative methylotrophic bacteria based on $5 S$ rRNA sequences. In Microbial Growth on $\mathrm{C}_{1}$ Compounds, pp. 275-284. Edited by J. C. Murrell \& D. P. Kelly. Andover: Intercept.

Delwiche, C. F. \& Palmer, J. D. (1996). Rampant horizontal transfer and duplication of RubisCO genes in eubacteria and plastids. Mol Biol Evol 13, 873-882.

Dickerson, R. E. (1980). Evolution and gene transfer in purple photosynthetic bacteria. Nature 283, 210-212.

Felsenstein, J. (1989). PHYLIP - phylogeny inference package (version 3.2). Cladistics 5, 164-166.

Fonstein, M., Koshy, E. G., Nikolskaya, T., Mourachov, P. \& Haselkorn, R. (1993). Refinement of the high resolution physical and genetic map of Rhodobacter capsulatus and genome surveys using blots of the cosmid encyclopedia. EMBO J 14, 1827-1841.

Gibson, J. L. (1995). Genetic analysis of $\mathrm{CO}_{2}$ fixation genes. In Anoxygenic Photosynthetic Bacteria, pp. 1107-1124. Edited by R. E. Blankenship, M. T. Madigan \& C. E. Bauer. Dordrecht: Kluwer.

Gibson, J. L. \& Tabita, F. R. (1977a). Different molecular forms of D-ribulose-1,5-bisphosphate carboxylase from Rhodopseudomonas sphaeroides. J Biol Chem 252, 943-949.

Gibson, J. L. \& Tabita, F. R. (1977b). Isolation and preliminary characterization of two forms of ribulose 1,5-bisphosphate carboxylase from Rhodopseudomonas capsulata. J Bacteriol 132, 818-823.

Gibson, J. L. \& Tabita, F. R. (1993). Nucleotide sequence and functional analysis of CbbR, a positive regulator of the Calvin cycle operons of Rhodobacter sphaeroides. J Bacteriol 175, 5778-5784.

Gibson, J. L. \& Tabita, F. R. (1996). The molecular regulation of the reductive pentose phosphate pathway in proteobacteria and cyanobacteria. Arch Microbiol 166, 141-150.

Goethals, K., van Montagu, M. \& Holsters, M. (1992). Conserved motifs in a divergent nod box of Azorhizobium caulinodans ORS571 reveal a common structure in promoters regulated by LysR-type proteins. Proc Natl Acad Sci USA 89, 1646-1650.

Igarashi, Y. \& Kodama, T. (1996). Genes related to carbon dioxide fixation in Hydrogenovibrio marinus and Pseudomonas hydrogenothermophila. In Microbial Growth on $C_{1}$ Compounds, pp. 88-93. Edited by M. E. Lidstrom \& F. R. Tabita. Dordrecht: Kluwer.

Imhoff, J. F., Truper, H. G. \& Pfennig, N. (1984). Rearrangement of the species and genera of the phototrophic 'purple nonsulfur bacteria'. Int J Syst Bacteriol 34, 340-343.

Jungst, A. \& Zumft, W. G. (1992). Interdependence of respiratory NO reduction and nitrite reduction revealed by mutagenesis of nirQ, a novel gene in the denitrification gene cluster of Pseudomonas stutzeri. FEBS Lett 314, 308-314.

Kusano, T. \& Sugawara, K. (1993). Specific binding of Thiobacillus ferrooxidans $\mathrm{RbcR}$ to the intergenic sequence between the $r b c$ operon and the rbcR gene. J Bacteriol 175, 1019-1025.

Kutsche, M., Leimkuhler, S., Angermuller, S. \& Klipp, W. (1996). Promoters controlling expression of the alternative nitrogenase and the molybdenum uptake system in Rhodobacter capsulatus are activated by $\mathrm{NtrC}$, independent of $\sigma^{54}$, and repressed by molybdenum. J Bacteriol 178, 2010-2017.

Larimer, F. W., Lu, T.-Y. S. \& Buley, D. M. (1995). Sequence and expression of the form II ribulose bisphosphate carboxylase/ oxygenase (RUBISCO) gene from Rhodobacter capsulatus. FASEB J 9, A1275.

Lorbach, S. C. \& Shively, J. M. (1995). Identification, isolation, and sequencing of the ribulose bisphosphate carboxylase/oxygenase regulatory genes ( $c b b R I$ and $c b b R I I)$ in Thiobacillus denitrificans. In Abstracts of the $95^{\text {th }}$ General Meeting of the American Society for Microbiology, p. 528. Washington, DC: American Society for Microbiology. 
Ludwig, W., Rosello-Mora, R., Aznar, R. \& 14 other authors (1995). Comparative sequence analysis of $23 \mathrm{~S}$ rRNA from proteobacteria. Syst Appl Microbiol 18, 164-188.

Meijer, W. G., van den Bergh, E. R. E. \& Smith, L. M. (1996). Induction of the gap-pgk operon encoding glyceraldehyde-3phosphate dehydrogenase and 3-phosphoglycerate kinase of Xanthobacter flavus requires the LysR-type transcriptional activator CbbR. J Bacteriol 178, 881-887.

Paoli, G. C., Strom-Morgan, N., Shively, J. M. \& Tabita, F. R. (1995). Expression of the $c b b L c b b S$ and $c b b M$ genes and distinct organization of the $c b b$ Calvin cycle structural genes of Rhodobacter capsulatus. Arch Microbiol 164, 396-405.

Pridmore, R. D. (1987). New and versatile cloning vectors with kanamycin-resistance marker. Gene 56, 309-312.

Schell, M. A. (1993). Molecular biology of the LysR family of transcriptional regulators. Annu Rev Microbiol 47, 597-626.

Scolnik, P. A. \& Haselkorn, R. (1984). Activation of extra copies of genes coding for nitrogenase in Rhodopseudomonas capsulata. Nature 307, 289-292.

Strimmer, K. \& von Haeseler, A. (1996). Quartet puzzling: a quartet maximum likelihood method for reconstructing tree topologies. Mol Biol Evol 13, 964-969.

Thompson, J. D., Higgins, D. G. \& Gibson, T. J. (1994). CLustaL w : improving the sensitivity of progressive multiple sequence alignment through sequence weighting, position-specific gap penalties and weight matrix choice. Nucleic Acids Res 22, 4673-4680.

Viale, A. M., Kobayashi, H., Akazawa, T. \& Henikoff, S. (1991). $r b c R$, a gene coding for a member of the LysR family of transcriptional regulators, is located upstream of the expressed set of ribulose 1,5-bisphosphate carboxylase/oxygenase genes in the photosynthetic bacterium Chromatium vinosum. J Bacteriol 173, 5224-5229.

Walker, J. E., Saraste, M., Runswick, M. J. \& Gay, N. J. (1982). Distantly related sequences in the alpha- and beta-subunits of ATP synthase, myosin, kinases and other ATP-requiring enzymes and a common nucleotide binding fold. EMBO J 1, 945-951.

Watson, G. M. F. \& Tabita, F. R. (1996). Regulation, unique organization, and unusual primary structure of carbon fixation genes from a marine phycoerythrin-containing cyanobacterium. Plant Mol Biol 32, 1103-1115.

Watson, G. M. F. \& Tabita, F. R. (1997). Microbial ribulose 1,5bisphosphate carboxylase/oxygenase: a molecule for phylogenetic and enzymological investigation. FEMS Microbiol Lett 146, 13-22.

Windhovel, U. \& Bowien, B. (1991). Identification of $c f x R$, an activator gene of autotrophic $\mathrm{O}_{2}$ fixation in Alcaligenes eutrophus. Mol Microbiol 5, 2695-2705.

Woese, C. R., Gibson, J. \& Fox, G. E. (1980). Do genealogical patterns in purple photosynthetic bacteria reflect interspecific gene transfer? Nature 283, 212-214.

Woese, C. R., Stackebrandt, E., Weisburg, W. G. \& 8 other authors (1984). The phylogeny of purple bacteria: the alpha subdivision. Syst Appl Microbiol 5, 315-326.

Yokoyama, K., Hayashi, N. R., Arai, H., Chung, S. Y., Igarashi, Y. \& Kodama, T. (1995). Genes encoding RubisCO in Pseudomonas bydrogenothermophila are followed by a novel $c b b Q$ gene similar to nirQ of the denitrification gene cluster from Pseudomonas species. Gene 153, 75-79.

Received 16 May 1997; revised 14 August 1997; accepted 26 August 1997. 